\title{
College Students' Perception of Vulnerability/Susceptibility and Desire for Health Information
}

\author{
Susan Boehm, $\mathrm{PhD}^{\mathrm{a}}$, Esther J. Selves, MS ${ }^{\mathrm{a}}$, Edith Raleigh, $\mathrm{PhD}^{\mathrm{a}}$, David Ronis, $\mathrm{PhD}^{\mathrm{b}}$, \\ Patricia M. Butler, $\mathrm{PhD}^{\mathrm{c}}$ and Margaret Jacobs, $\mathrm{MS}^{\mathrm{d}}$
}

${ }^{a}$ School of Nursing, University of Michigan, Room 2179, ${ }^{b}$ Institute for Social Research. University of Michigan and Department of Veterans' Affairs, 'Ambulatory Care Nursing, University of Michigan Hospital and 'Student Health Services, University of Michigan $($ LSA

\begin{abstract}
The specific aims of this study were to develop a questionnaire and conduct a survey of college students 18-22 years of age, in order to determine (1) their perception of being vulnerable and susceptible to health problems and (2) their interest in receiving help and information about health maintenance. Material from four 2 -h focus groups provided the content for the initial questionnaire. Pilot testing of the early drafts consisted of several phases in which students completed the tool in both group situations and in face-to-face interviews. The final questionnaire was completed by 364 students on two campuses in the Midwest. Two scales were identified using factor analysis and analysis for internal consistency. The Vulnerability/ Susceptibility Scale demonstrated a good reliability (Kuder-Richardson-20 $=0.8136$ ) and five factors were identified through factor analysis. The Information Scale demonstrated a high reliability (Kuder-Richardson-20 $=0.8958$ ) and two factors were identified through factor analysis. Based on analysis of variance (ANOVA) data, a model was developed using the variables of the Vulnerability/Susceptibility Scale, the Information Scale, gender and health rating. The model was then tested using multiple regression analysis. This study identified health related areas about which
\end{abstract}

the students feel vulnerable/susceptible and are interested in receiving health help/information. This study suggests which population of college students might be most receptive to health information and future behavioral strategies.

Key words: Behavioral model; College students; Vulnerability; Health Information.

\section{Introduction}

It has been suggested that the principles and procedures of behavioral change developed by experimental psychologists over the last twenty years may be useful for learning new health habits and for changing unhealthy habits [1,2]. This is of particular importance given that research has demonstrated the need for Americans to change dietary habits and life-style patterns in order to reduce risks and maintain health.

There is also evidence that suggests teaching the young adult behavioral strategies for the self-management of health maintenance and care of minor illnesses has a potential outcome that is twofold $[3,4]$. First, the young adult may be able to alter unhealthy habits at a younger age and thereby 
reduce the accumulated risks of disease. Secondly, they may be able to learn and practice behavioral strategies that will be applicable as they move into the middle years.

However, the young adult population has not been of widespread interest to those researchers and clinicians interested in behavior change in order to maintain health [5]. The lack of health focused research among this age group (18-22) is related to the fact that young adults are usually at optimal health and developmentally perceive themselves to be invulnerable to illness $[4,6]$.

There have been a number of research recommendations for this age group as a consequence of previous studies. Such recommendations include the need to evaluate the students' perception of the extent to which they are at risk and their willingness to undertake change [7]. Another recommendation is that the students need to indicate the extent to which they are willing to participate in a program, including how, where and when the students would be involved [7]. Finally, the program should recognize the need for tailoring the intervention relative to the specific information the student wants and the profile of the student [8].

The specific aims of this study were to develop an instrument and conduct a survey of college students 18-22 years of age, in order to determine their perception of being vulnerable and their interest in receiving information about health. This survey was designed as the first step in the development of a behavioral self-management program that will emphasize self-management skills for the young adult aimed at health maintenance, primary risk-reduction and self-care of minor illness.

\section{Method}

Questionnaire de 'elopment

The questionnaire was developed, tested and implemented by a collaborative research team consisting of the authors and members from the Institute for Social Research at the University of Michigan. The process of questionnaire development included four 2-h focus groups with college students 18-22 years old. The material from the focus groups provided the content for the initial draft of the questionnaire. Pilot testing of the early drafts consisted of several phases in which students completed the tool in both group situations and in face-to-face interviews. After each phase the research team made revisions and refinements based on the students' reactions and preferred word choices. The final questionnaire was titled American Students." A Survey of Health and Health Behaviors.

This questionnaire was self-administered, taking 15-20 min to complete. It was accompanied by a cover letter explaining the purpose of the study, informed consent information and the mechanism for obtaining a summary of the data gathered. No names were requested and anonymity was further ensured by asking the subjects to seal their questionnaires in the envelopes provided prior to returning them to the investigators. Informed consent was assumed by completion of the questionnaire.

\section{Sample}

The sample reported here consisted of 364 students on two campuses in the Midwest. There were more females $(59 \%)$ than males $(41 \%)$ and more who were not employed $(57 \%)$ than employed $(43 \%)$ while attending classes. The sample was well distributed across class rank with the exception of a slightly higher percentage of sophomores $(29 \%)$ than either freshpersons $(24 \%)$, juniors $(24 \%)$, or seniors $(22 \%)$. One percent of the subjects was graduate students. Nearly all of the students were never married $(99 \%)$. The majority of students lived either in the dormitory $(51.6 \%)$, off campus with a roommate $(27.7 \%)$, or in a fraternity or sorority $(17.6 \%)$, with the remaining students $(3.1 \%)$ having a variety of other living arrangements. A ma- 
jority of the students identified themselves as having some religious background $(37 \%$ Catholic, 33\% Protestant, 18\% Jewish, 10\% none, $2 \%$ other). Finally, $92.6 \%$ of the students were white $(4.1 \%$ black, $0.3 \%$ Hispanic, $2.5 \%$ Asian and $0.5 \%$ other).

In terms of self-rated health, 154 students $(42.5 \%)$ rated their health as excellent, 121 $(33.4 \%)$ as above average, $73(20.2 \%)$ as average, $13(3.6 \%)$ as below average, and 1 $(0.3 \%)$ as poor.

\section{Development of scales}

The two scales used in this study (ulnerability/susceptibility and information) were identified using analysis for internal consistency and principle components analysis.

Vulnerability/Susceptibility Scale. The researchers recognized that, developmentally, individuals from 18-22 years of age tend to believe themselves to be invulnerable. Thus, the questionnaire was designed to determine if the respondents might perceive themselves to be among the 'worried well' and at least marginally aware of their vulnerability as a result of some of their behaviors $[9,7]$.

This scale was built on 30 dichotomous items which asked the students about specific health issues they experienced in the past 6 months and whether they expected such cxperiences in the next 12 months. The original list of items was gleaned from surveys reported in the literature and were reduced or altered following the focus groups and interviews of students who completed pilot versions of the questionnaire. The list included such items as changes in health, eating or sleep; incidents of depression or sexual difficulties; experiences with major or minor ilInesses, accidents, or stress; and reason to have a physical exam or visit the doctor or health services.

Maximum likelihood extraction with varimax rotation was performed through SPSSx on 18 items composing the Vulnerability/ Susceptibility Scale. Principle components extraction on the original 30 items was used prior to maximum likelihood extraction to estimate number of factors, presence of outliers, absence of multicolinearity and factorability of the correlation matrices. Twelve of the items were identified as outliers and not well-defined by the factor solution as they did not load on any factor. These items pertained to sexual difficulties, pregnancy, major illness, minor accidents and physical exams. The remaining items loaded on five factors accounting for $62 \%$ of the variance. Simple structure was achieved by this factor solution. The eigenvalues ranged from 1.40 to 4.56 . These factors were labeled as follows: Basic Needs, Major Stress, Minor Illness, Diet and Minor Stress. The factor loadings are displayed in Table 1. Even though Diet is a trivial factor, the two variables are highly correlated with each other $(r=0.73)$ and relatively uncorrelated with other variables $(r<0.16)$, indicating the factor may be reliable [10].

Table 1. Factor loadings ${ }^{\text {a }}$ for the Vulnerability/ Susceptibility Scale.

\begin{tabular}{|c|c|c|c|c|}
\hline Item & F1 & $\mathrm{F} 2 \quad \mathrm{~F} 3$ & $\mathrm{~F} 4$ & F5 \\
\hline Expect change in sleep & 0.82 & & & \\
\hline Past change in sleep & 0.80 & & & \\
\hline Expect eating change & 0.70 & & & \\
\hline Past eating change & 0.69 & & & \\
\hline Expect depression & & 0.81 & & \\
\hline Past depression & & 0.77 & & \\
\hline Expect major stress & & 0.72 & & \\
\hline Past major stress & & 0.67 & & \\
\hline Past minor illness & & 0.73 & & \\
\hline Expect to visit $\mathrm{MD}$ & & 0.68 & & \\
\hline Expect minor illness & & 0.67 & & \\
\hline Past visit to $\mathrm{MD}$ & & 0.66 & & \\
\hline Expect to diet & & & 0.92 & \\
\hline Past diet & & & 0.90 & \\
\hline Expect minor stress & & & & 0.67 \\
\hline Past minor stress & & & & 0.63 \\
\hline Past health change & & & & -0.55 \\
\hline Expect health change & & & & -0.54 \\
\hline
\end{tabular}

${ }^{\mathrm{a}}$ Factor loadings $<0.40$ were omitted. 
The scores on the Vulnerability/Susceptibility Scale had a possible range of $18-36$, with higher scores reflecting less vulnerability/susceptibility. The mean score for this sample was 27.4 (range $=18-36$, S.D. $=4.1$ ). Analysis of the Vulnerability/Susceptibility Scale demonstrated a good reliability (KuderRichardson- $20=0.8136$ ).

Information Scale. A second highly reliable scale was also developed concerning the students' interest in obtaining help/information regarding health (Kuder-Richardson$20=0.8958$ ). This scale was based on 14 dichotomous items that asked the students to indicate their interest in receiving help or information on items such as diet, practicing good health habits, stress management, selfcare for minor illness and how to stay healthy for the middle years.

Principle components analysis with varimax rotation was performed through SPSSx on these 14 items to estimate number of factors, presence of outliers, absence of multicolinearity and factorability of the correlation matrices. Since there were no outliers and no multicolinearity, maximum likelihood extraction was performed. Two factors were extracted which accounted for $54 \%$ of the variance. Simple structure was achieved by this factor solution. The eigenvalues were 5.98 and 1.58. These factors were labeled as follows: General Health and Sexual Activity. The factor loadings are displayed in Table 2.

The possible scores on this scale ranged from 14 to 28 , with higher scores reflecting less desire for information. The mean score for this sample was 20.831 (range $=14-28$, S.D. $=4.4839$ ).

\section{Results}

\section{Model development}

In order to determine what variables may be related to the Vulnerability/Susceptibility Scale and the Information Scale, analysis of variance (ANOVA) was performed using
Table 2. Factor loadings" for the Information Scale.

\begin{tabular}{lll}
\hline Item & F1 & F2 \\
\hline Practicing good health habits & 0.79 \\
Nutrition & 0.78 \\
How to stay healthy now & 0.75 \\
Exercise & 0.70 \\
How to stay healthy in middle years & 0.67 \\
Self-care for minor illness & 0.59 \\
Stress management & 0.54 \\
Dieting & 0.53 \\
Accident prevention & 0.50 \\
Safe sex & \multicolumn{2}{c}{0.85} \\
Sexually transmitted diseases & \multicolumn{2}{c}{0.81} \\
Birth control & & 0.77 \\
AIDS & & 0.76 \\
Problem pregnancy & 0.59
\end{tabular}

${ }^{\mathrm{a}}$ Factor loadings $<0.40$ were omitted.

several of the descriptive variables. These variables included gender, class, age, race, living arrangements, marital status, participation in a monogamous sexual relationship, employment status, full or part-time student status and self-rated health.

For the Vulnerability/Susceptibility Scale, where higher scores reflect less vulnerability/susceptibility, females $(M=26.1)$ rated themselves as more vulnerable/susceptible than did the males $(M=29.3)$ in the sample $[F$ $(1,323)=53.961, P<0.001]$. Also, there were significant differences on the Vulnerability/Susceptibility Scale depending on self-rated health $[F(4,318)=9.815, P<$ $0.001]$. The mean scores progressed in a linear fashion with those rating themselves as below average or poor in health also rating themselves as more vulnerable/susceptible (poor $=22.0$, below average $=23.6$, average $=$ 26.1 , above average $=26.9$ and excellent $=$ 28.8). With the exception of the one person who gave a poor health rating, significant differences were demonstrated in all but one of 
the non-simultaneous pairwise comparisons of health ratings $(P<0.05)$. Those who rated their health as above average did not have significantly different scores on this scale from those who rated their health as average.

For the Information Scale, where higher scores reflect a desire for less information, females $(M=19.4)$ wanted significantly more information than did males $(M=22.9)$ in the sample $[F(1,342)=57.437, P<0.001]$. Also significant differences in the Information Scale were detected based on self-rated health $[F(4,337)=3.991, P<0.01)$. In the nonsimultaneous pairwise comparisons, significant differences were detected between those who rated their health as above average $(M=19.61)$ and both those who rated their health as excellent $(M=21.74, P<0.001)$ and those who rated their health as average $(M=21.06, P<0.05)$. No other pairwise comparisons demonstrated significant differences. (The mean score on the Information Scale for those who rated their health as below average was 20.77 . The person who's health rating was poor had a 17.00 on the Information Scale.)

Based on the analysis of variance data, the preliminary information model depicted in Fig. 1 was constructed to explain the variance in the scores on the Information Scale. The ANOVAs demonstrated that both gender and self-rated health were associated with significant differences on both scales. Therefore, direct relationships were postulated between gender and each scale and self-rated health and each scale. The direct relationship between the Vulnerability/ Susceptibility Scale and the Information Scale was postulated on the theoretical basis that those who perceive themselves as more vulnerable/susceptible to health problems will desire more health information.

This final relationship is consistent with other theories of health behavior and decision-making. Contingency models of decision-making postulate that the importance and relevance of a problem to an in-
Fig. 1. Sequence of recruitment, measurement.

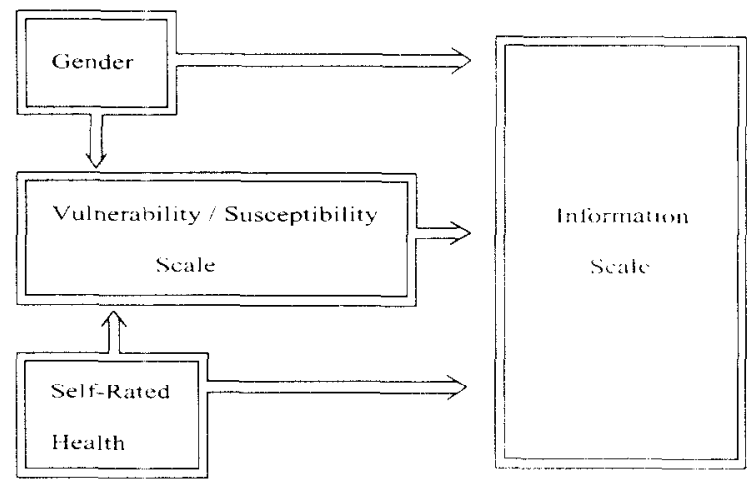

dividual influence the extent of the decision-making process $[11,12]$. If a threat is perceived as sufficiently serious and relevant, an active decision must be made about it. This decision will require information gathering to search for potential solutions [12]. Within the Health Belief Model [13] perceived susceptibility is one predictor of health behavior. It may be argued that desire for health information is an intermediary step between perceived vulnerability/susceptibility and the initiation of health behavior.

The first portion of the preliminary information model was tested by regressing the Vulnerability/Susceptibility Scale on gender and self-rated health. The results of this analysis are presented in Table 3 . This analysis demonstrates that both gender and self-rated health were significantly linearly related to the Vulnerability/Susceptibility Scale.

Multiple regression was also used to test the entire model. The Information Scale was regressed on all three independent variables, with gender and self-rated health being entered simultaneously in the first step, followed by the Vulnerability/Susceptibility Scale in the second step. The results of this analysis are presented in Table 4 . This final multiple regression analysis demonstrated 
Table 3. Regression of Vulnerability/Susceptibility Scale on gender and health rating.

\begin{tabular}{lllll}
\hline & $b$-Value & S.E. & $t$-Value & $P$-value \\
\hline $\begin{array}{l}\text { Gender } \\
\text { Health }\end{array}$ & -0.45804 & 0.08492 & -5.3941 & $<0.001$ \\
Rating & -0.21969 & 0.04767 & -4.6082 & $<0.001$ \\
\hline$N=317, R=0.40885, R^{2}=0.16716, F(2,314)=$ \\
$\begin{array}{l}\text { 31.512, } P<0.001 .\end{array}$
\end{tabular}

that the Vulnerability/Susceptibility Scale added $4.3 \%$ explained variance in the Information Scale over and above gender and selfrated health.

Because self-rated health was nonsignificant in the previous multiple regression of the Information Scale on the independent variables, a single regression of the Information Scale on self-rated health was performed. This single regression was also nonsignificant $[F$ $(1,341)=3.0652, P>0.05)$. Therefore, the direct relationship between self-rated health and the Information Scale was deleted from the model, while the indirect relationship between self-rated health and the Information Scale through the Vulnerability/Susceptibility Scale was retained. This indirect relationship
Fig. 2. Preliminary information model.

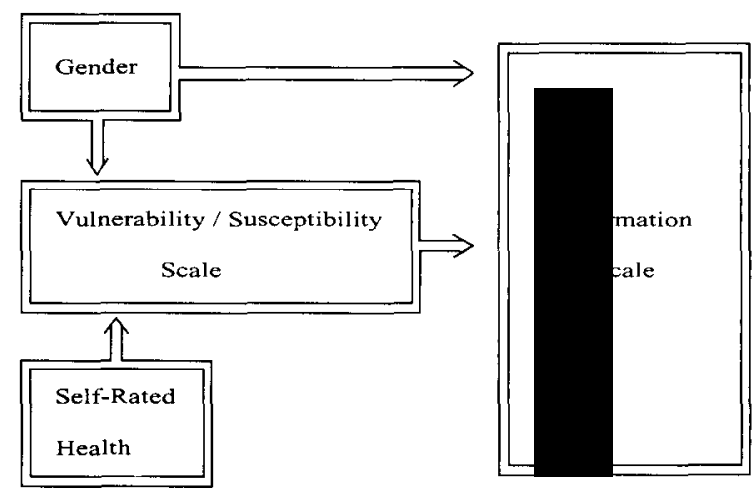

was retained due to the strong linear relationship between self-rated health and the Vulnerability/Susceptibility Scale. Thus, the final information model was formulated as depicted in Fig. 2.

As another test of its validity, the final information model was also subjected to significance tests of the two following null hypotheses: (a) there are no differences among the four classes in the regression equation (including the coefficients) predicting vulnerability/susceptibility and (b) there are no differences among the four classes in the regression equation (including the coeffi-

Table 4. Selected ordered regression of Information Scale on gender, health rating and Vulnerability/Susceptibility Scale.

\begin{tabular}{|c|c|c|c|c|c|}
\hline & $r$-Value & $b$-Value & S.E. & $t$-Value & $P$-Value \\
\hline \multicolumn{6}{|l|}{ Step $1^{\mathrm{a}}$} \\
\hline Gender & -0.35287 & -3.2618 & 0.49524 & -6.5862 & $<0.001$ \\
\hline Health Rating & -0.00627 & -0.02997 & 0.27373 & -0.10950 & NS \\
\hline \multicolumn{6}{|l|}{ Step $2^{b}$} \\
\hline Gender & -0.27393 & -2.5575 & 0.51498 & -4.9661 & $<0.001$ \\
\hline Health Rating & 0.05381 & 0.26035 & 0.27709 & 0.9396 & NS \\
\hline Vul/Susc Scale & 0.22252 & 0.25242 & 0.06343 & 3.9796 & $<0.001$ \\
\hline
\end{tabular}

aor Step 1: $N=308, R=0.36056, R 2=0.13000, F(2,305)=22.788, P<0.001$.

${ }^{\mathrm{b}}$ For Step 2: $N=308, R=0.41603, R 2=0.17308, F(3,304)=21.210, P<0.001$. 
cients) predicting desire for health information. To accomplish these significance tests, two regression models were estimated for both the first portion of the final information model and for the entire final information model, using the standard method for testing whether regression models differ across subgroups [14].

First, four sets of new variables were created. The first set was dummy variables of the freshperson, sophomore, and junior classes. The second, third and fourth sets were these dummy variables multiplied by gender, by self-rated health and by vulnerability/susceptibility scores, respectively.

The first portion of the final information model was then tested by performing a multiple regression analysis of vulnerability/susceptibility on gender, sclf-rated health, and the three dummy class variables $[F(5,314)=$ $16.381, P<0.001$ ). A second multiple regression analysis of vulnerability/susceptibility on gender, self-rated health, the dummy class variables, the gender-by-class variables, and the self-rated health-by-class variables was also performed $[F(11,308)=7.7418, P<$ $0.001)$.

The question to be answered at this point was whether the gender-by-class variables and the self-rated health-by-class variables as a group add significantly to the prediction over and above gender, self-rated health and the three dummy class variables. To answer this question, an $F$-statistic was calculated to determine if the sum of squares of the second multiple regression analysis was signiticantly greater than that for the first test of the first portion of the model $[F(6,308)=0.08232$, $P>0.05]$. This finding means that vulnerability/susceptibility could not be more accurately predicted by using different regression equations for each of the four classes.

The entire information model was then subjected to similar analyses, first regressing the Information Scale on gender, self-rated health, the Vulnerability/Susceptibility Scale and the three dummy class variables [ $F$ $(6,299)=12.697, P<0.001]$ and secondly regressing the Information Scale on gender, self-rated health, the Vulnerability/ Susceptibility Scale, the dummy class variables, the gender-by-class variables, the self-rated health-by-class variables and the vulnerability/susceptibility-by-class variables $[F(15,290)$ $=5.9759, P<0.001)$. Finally, an $F$-statistic was calculated to determine if the sum of squares of the second multiple regression was significantly greater than that for the first test of the entire information model $[F(9,290)$ $=0.23330, P>0.05]$. This indicates that interest in health information could not have been more accurately predicted by using different regression equations for each of the four classes. In summary, there was no evidence of a need to modify the model to apply to different classes.

Table 5. Percentages of students interested in receiving health help/information by topic.

\begin{tabular}{ll}
\hline Topic & $\begin{array}{l}\text { Percent } \\
\text { desiring help/ } \\
\text { information }\end{array}$ \\
\hline How to stay healthy now & 63 \\
Exercise & 62 \\
Nutrition & 60 \\
Stress management & 60 \\
Good health habits & 56 \\
How to stay healthy in middle & \\
$\quad$ years & 56 \\
Self-care for minor illness & 51 \\
Dieting & 44 \\
AIDS & 59 \\
Sexually transmitted disease & \\
$\quad$ (STD) & 54 \\
Birth control & 52 \\
Safe sex & 49 \\
Problem pregnancy & 28 \\
Accident prevention & 21.5 \\
\hline
\end{tabular}




\section{Health information topics}

The students were asked to indicate, from among 14 topics, those topics about which they would be interested in receiving help or information. The topics included eight items related to health, five related to sexual activity, and one related to accidents. If they chose a topic, they were then asked to choose, from four sites, at which site(s) they would prefer to receive the help or information. They could select all that applied. The sites included Student Health Services, the dormitory, the recreation building and the Campus Information Center. If they chose a topic they were also asked to indicate by which of three methods they would prefer to receive the information: one-to-one, groups, or reading materials. Again, they could select all that applied. The results of these questions are presented in Tables 5-7.

Results indicated that most of the students desired help or information about all of the topics except dieting, safe sex, problem pregnancy and accident prevention. Particularly low percentages of students were interested in information on the last two topics.

In general, the preferred location for receiving health help or information was at the student health service, with the dormitory as the second most preferred location. For exercise, however, the recreation building was the most preferred location.

For all topics, most subjects preferred reading material over group or one-to-one presentation. Group presentation was second most preferred for most topics; however, for information about dieting and many sexrelated topics (birth control, safe sex and problem pregnancy) one to-one presentation was the second most preferred medium.

The students were also asked to indicate, from a list of five obstacles, the main obstacles to their seeking help/information for the

Table 6. Preferred locations for receiving health help/information.

\begin{tabular}{lllll}
\hline Topic & \multicolumn{2}{l}{ Percent preferring location } & \\
\cline { 2 - 5 } & $\begin{array}{l}\text { Student } \\
\text { health } \\
\text { service }\end{array}$ & Dormitory & $\begin{array}{l}\text { Recreation } \\
\text { building }\end{array}$ & $\begin{array}{l}\text { Campus } \\
\text { information } \\
\text { center }\end{array}$ \\
\hline How to stay healthy now & 56 & & & 19 \\
Exercise & 27 & 34 & 44 & 18 \\
Nutrition & 50 & 30 & 13 & 19 \\
Stress management & 46 & 42 & 8 & 28 \\
Good health habits & 52 & 31 & 12.5 & 18 \\
How to stay healthy in & 61 & 30 & 11 & 20 \\
middle years & & & & 18 \\
Self-care for minor illness & 62.5 & 27 & 6 & 19 \\
Dieting & 48 & 34 & 8 & 23 \\
AIDS & 62 & 33 & 7 & 18 \\
STD & 65 & 30 & 4 & 18 \\
Birth control & 69 & 26 & 5 & 20 \\
Safe sex & 68 & 31 & 5 & 26 \\
Problem pregnancy & 67 & 28 & 9 & \\
Accident prevention & 51 & 42 & & 14 \\
\hline
\end{tabular}


Table 7. Preferred method of receiving health help/information.

\begin{tabular}{llll}
\hline Topic & \multicolumn{2}{l}{ Percent preferring method } \\
\cline { 2 - 3 } & One to one & Group & Reading material \\
\hline How to stay healthy now & 20 & 30 & 65 \\
Exercise & 24 & 39 & 54 \\
Nutrition & 25 & 29 & 63 \\
Stress management & 34 & 39 & 53 \\
Good health habits & 22 & 32 & 61 \\
How to stay healthy in middle years & 18 & 29 & 70 \\
Self-care for minor illness & 19 & 31 & 65 \\
Dieting & 33 & 22 & 62.5 \\
AIDS & 26 & 40 & 62 \\
STD & 28 & 29 & 64 \\
Birth control & 44 & 22 & 57 \\
Safe sex & 36 & 30 & 59 \\
Problem pregnancy & 49 & 23 & 59 \\
Accident prevention & 18 & 45 & 68 \\
\hline
\end{tabular}

above topics. They could choose all that applied to their circumstances. Sixty-eight percent $(68 \%)$ indicated time as an obstacle with $33 \%$ indicating inconvenient time, $29 \%$ indicating inconvenient place and $25 \%$ indicating uncomfortable setting. Only 13\% indicated that money was an obstacle. Twenty percent $(20 \%)$ listed various other obstacles to seeking health information. These other obstacles included not being motivated to seek information, knowing all that was needed, an uncomfortable topic and not worrying about health. A small number of students $(2 \%)$ responded that there were no obstacles to seeking health information.

\section{Discussion}

\section{Model development}

A major contribution of this research is that it not only determined the perception of vulnerability/ susceptibility and the interest in health information of college students, but it also proposed a model for identifying those college students who would be more likely to be interested in health information based on their gender, self-rated health and Vulnerability/ Susceptibility score. This model has validity independent of class level. This is important because one purpose of this research was to identify future directions for interven-

Table 8. Practice implications.

- Design marketing approaches and interventions to overcome perception of invulnerability

- Provide health related information and assistance in locations students prefer

- Identify unique needs based on genders, age and topic

- Be sensitive to time constraints and pressure 
ing in this population in order to increase behaviors that would serve to keep tile students healthy now, as well as provide a model of behavior to keep them healthy in the future. Although it would be inefficient to determine the self-rated health and the Vulnerability/ Susceptibility score for all incoming freshpersons, these tools may be useful in further narrowing a sample to be used in an intervention study. By identifying those students most likely to want health information, it may be possible to maximize the effects of any future interventions. It is also evident from this study that targeting females for future interventions related to health help/information would likely have greater impact than targeting males.

\section{Health information topics}

Interest in receiving help/information on health related topics was not surprising. The focus group discussions provided a sense that health related topics for their present years, as well as for their middle years, held significant importance among themselves and their peer group. These focus group discussions revealed that their interest in health was an important aspect of their image and it was not necessarily related to health maintenance. More than one student expressed concern that they did not want to repeat the unhealthy practices of their parents and the generations immediately ahead of them. Often these points were made in conjunction with general concerns over the environment and 'greed' identified with the 1980s. As a group, however, they demonstrated the expected sense of invulnerability of the age group as evidenced by the few $(21.5 \%)$ who identified an interest in receiving help/ information on accident prevention.

\section{Implications for future research}

The intent of this research was to explore the potential for a behavioral intervention that would assist the college student in the learning and practice of behaviors for the maintenance of health and management of minor illnesses. The findings from this study would suggest that there are students who indicate an interest in learning more about such behaviors. Their motivations are not clear, but may be related to concerns over their health as much as to current emphasis on health by peers and society. Thus, the current emphasis on health might provide a window of time for the testing of interventions for this age group that could be adjusted at a future time when the emphasis an health is not as popular. Such an intervention would surely be strengthened by frequent and visible opportunities for peer and social reinforcement.

While the majority of the students indicated that they preferred the help/information via reading material, the research team senses that this would not be the most effective method. The time constraints and study pressures would suggest that the intervention needs to be designed to be very efficient and very creative. The academic setting is very competitive, requiring considerable focused attention by the student. In addition, most students are required to work and/or demonstrate some degree of extra curricular activity. Thus, a behavioral intervention needs to be such that it will be integrated into the students' demanding life styles.

\section{Conclusions}

This study has yielded a model for use in identifying students who would be most likely to be interested in health help/information. It has also revealed that the health information topic determines how and where the students want help/information. Finally, the data from this study can provide a beginning for developing a behavioral self-management program for college students.

\section{Acknowledgments}

Support for this study was obtained from Biomedical Research, University of Michigan School of Nursing. 


\section{References}

1 Foryet JD et al.: Plasma lipid changes in the normal population following behavioral treatment. $\mathbf{J}$ Consult Psychol 1979; 47: 440-452.

2 Wilson HM et al:: Behavior therapy for obesity: An evaluation of treatment and outcome. Adv Behav Res Ther 1980; 3: 49-86.

3 Johnson-Saylor M: Seize the moment: health promotion for the young adult. Top Clin Nurs 1980; 2(2): 9-19.

4 Lipnickey SC: The potential for formulating a healthy lifestyle: An assessment of health instruction and the university student. Eval Health Profess 1986; 9: 487-506.

5 Hovell MF et al.: Risk of excess weight gain in university women: A three-year community controlled analysis. Addict Behav 1985; 10: 15-28.

6 Newell-Winthrow C: Identifying health-seeking behaviors: a study of adolescents. Adolescence 1986; 21: 641-658.

7 Cormier DS: Lifestyle change on the campus: Pilot test of a program to improve student health practices. In: Davidson $\mathrm{P}$ and Davidson $\mathrm{S}$, editors. Behavioral medicine: changing health life styles. New York: Brunner/Mazel, 1960.
8 Bensley LB: Health risk appraisals in teaching health education in colleges and universities. Health Educ 1981; $12(6): 31-33$

9 Gochman DS: The organizing role of motivation in health beliefs and intentions. J Health Soc Behav 1972; 13 : 285-293.

10 Tabachnick B et al.: Using Multivariate Statistics, 2nd ed. New York: Harper \& Row, 1989.

11 Beach LR et al.: A contingency model for the selection of decision strategies. Acad Manage Rev 1978; 3: 439-448.

12 Janis IL et al.: Decision making: a psychological analysis of conflict, choice and commitment. New York: Free Press, 1977.

13 Janz NK et al.: The health belief model: a decade later. Health Educ Q 1984; 11: 1-47.

14 Pedhazur EJ: Multiple regression in behavioral research: explanation and prediction, 2nd ed. New York: Holt. Reinhart and Winston, 1982.

\section{Correspondence to:}

Susan Boehm PhD, RN

University of Michigan, School of Nursing

Medical Surgical Nursing, 2179 Nursing Annex

400 North Ingals Ann Arbor, MI 48109-0482, USA 